\title{
Image Equalization Using Singular Value Decomposition and Discrete Wavelet Transform
}

\author{
Cagri Ozcinar ${ }^{1}$, Hasan Demirel ${ }^{2}$ and Gholamreza Anbarjafari ${ }^{3}$ \\ ${ }^{1}$ Department of Electronic Engineering, University of Surrey, \\ GU2 7XH Surrey \\ ${ }^{2}$ Department of Electrical and Electronic Engineering, \\ Eastern Mediterranean University, \\ Gazimagusa, KKTC, via Mersin 10 \\ ${ }^{3}$ Department of Information Systems Engineering, \\ Cyprus international University, \\ Lefkosa, KKTC, via Mersin 10 \\ ${ }^{1}$ England \\ 2,3Turkey
}

\section{Introduction}

Contrast enhancement is frequently referred as one of the most important issues in image processing. Contrast is created by the difference in luminance reflected from two adjacent surfaces. In other words, contrast is the difference in visual properties that makes an object distinguishable from other objects and the background. In visual perception, contrast is determined by the difference in the color and brightness of the object with other objects. Our visual system is more sensitive to contrast than absolute luminance; therefore, we can perceive the world similarly regardless of the considerable changes in illumination conditions.

If the contrast of an image is highly concentrated on a specific range, e.g. an image is very dark; the information may be lost in those areas which are excessively and uniformly concentrated. The problem is to optimize the contrast of an image in order to represent all the information in the input image. There have been several techniques to overcome this issue (Shadeed et al., 2003; Gonzales and Woods, 2007; Kim et al., 1998; Chitwong et al., 2002). One of the most frequently used techniques is general histogram equalization (GHE). After the introduction of GHE, researchers came out with better techniques such as local histogram equalization (LHE). However, the contrast issue is yet to be improved and even these days many researchers are proposing new techniques for image equalization. In this work, we are comparing our results with two state-of-art techniques, namely, dynamic histogram equalization (DHE) (Abdullah Al Wadud et al., 2007) and our previously introduced singular value equalization (SVE) (Demirel et al. ISCIS 2008).

As motioned before, in many image-processing applications, GHE technique is one of the simplest and most effective primitives for contrast enhancement (Kim and Yang, 2006), 
which attempts to produce an output histogram that is uniform (Weeks et al., 1999). One of the disadvantages of the GHE is that the information laid on the histogram or probability distribution function (PDF) of the image will be lost. Demirel and Anbarjafari showed that the PDF of face images can be used for face recognition (Demirel and Anbarjafari, IEEE Signal Processing Letter, 2008), hence preserving the shape of PDF of the image is of vital importance. Techniques such as DHE or SVE are preserving the general pattern of the PDF of an image. DHE is obtained from dynamic histogram specification (Sun et al., 2005) which generates the specified histogram dynamically from the input image. DHE algorithm works in the following way (Abdullah Al Wadud et al., 2007): Firstly, the locations of local minimums of the histogram are found and then the histogram is divided into several subhistograms based on those local minimums. Then the mean, $\mu$, and standard deviation, $\sigma$, for each sub-histogram is calculated. If gray levels(GLs) of having frequencies within $(\mu-\sigma)$ to $(\mu+\sigma)$ is more than an specific value, e.g. $68.3 \%$ of the total number of GLs of a subhistogram, then that sub-histogram can be considered as a normal distribution of frequencies and there is no dominating portion. But if it is less then that threshold value, the sub histogram splits again. Then weights for GL range of $i^{\text {th }}$ sub-histogram are calculated by the following equation (Abdullah Al Wadud et al., 2007):

$$
\text { weight }_{i}=\operatorname{span}_{i} \times\left(\log S_{i}\right) x
$$

where $\operatorname{span}_{i}$ is GL range of sub-histogram $i, S_{i}$ is summation of all histogram values of $i^{\text {th }}$ sub-histogram, and $x$ is a coefficient to control the strength of image contrast. Then the range which is the expansion value to determine how many times to expand its subhistogram is calculated by using the fallowing formula (Abdullah Al Wadud et al., 2007):

$$
\text { range }_{i}=\frac{\text { weight }_{i}}{\sum_{i=1}^{n} \text { weight }_{i}} *(L-1)
$$

where $L$ is total number of available GLs. Finally, GHE is applied for each sub-histogram. SVE (Demirel et al. ISCIS 2008; Demirel and Anbarjafari, IEEE Signal Processing Letter, 2008) technique is based on equalizing the singular value matrix obtained by singular value decomposition (SVD). SVD of an image, which can be interpreted as a matrix is written as follows:

$$
A=U_{A} \Sigma_{A} V_{A}^{T}
$$

where $U_{A}$ and $V_{A}$ are orthogonal square matrices known as hanger and aligner respectively, and $\Sigma_{A}$ matrix contains the sorted singular values on its main diagonal. The idea of using SVD for image equalization comes from this fact that $\Sigma_{A}$ contains the intensity information of the given image (Tian et al., 2003). The objective of SVE proposed by Demirel et al. (ISCIS 2008 ) is to equalize a low contrast image in such a way that the mean moves towards the neighborhood of 8-bit mean gray value 128 in the way that the general pattern of the PDF of the image is preserved.

In our earlier work (Demirel and Anbarjafari, IEEE Signal Processing Letter, 2008), where we introduced PDF based face recognition, singular value decomposition was used to deal with the illumination problem. The method uses the ratio of the largest singular value of the 
generated normalized matrix over a normalized image which can be calculated according to equation (4).

$$
\xi=\frac{\max \left(\Sigma_{N(\mu=0, \mathrm{var}=1)}\right)}{\max \left(\Sigma_{A}\right)}
$$

where $\Sigma_{N(\mu=0, \mathrm{var}=1)}$ is the singular value matrix of the synthetic intensity matrix. This coefficient can be used to regenerate an equalized image using equation (5).

$$
\Xi_{\text {equalized }_{A}}=U_{A}\left(\xi \Sigma_{A}\right) V_{A}^{T}
$$

where $\Xi_{\text {equalized }_{A}}$ is representing the equalized image $A$. This task is eliminating the illumination problem.

Nowadays, wavelets have been used quite frequently in image processing. It has been used for feature extraction (Wang and Chen, 2006), denoising (Starck et al., 2002), compression (Lamard et al., 2005), image equalization enhancement (Demirel et al., IEEE Geoscience and Remote Sensing Letter, 2010), and face recognition (Liu et al., 2007). The decomposition of images into different frequency ranges permits the isolation of the frequency components introduced by "intrinsic deformations" or "extrinsic factors" into certain subbands (Dai and Yan, 2007). This process results in isolating small changes in an image mainly in high frequency subband images. Hence discrete wavelet transform (DWT) is a suitable tool to be used for designing pose invariant face recognition system. The two-dimensional wavelet decomposition of an image is performed by applying the one-dimensional DWT along the rows of the image first, and then the results are decomposed along the columns. This operation results in four decomposed subband images refer to Low-Low (LL), Low-High (LH), High-Low (HL), and High-High (HH). The frequency components of those subband images cover the frequency components of the original image.

In this work, we have proposed a new method for image equalization which is an extension of SVE and it is based on SVD of LL subband image obtained by DWT. DWT is used to separate the input image into different frequency subbands, where LL subband concentrates the illumination information. That is why, only LL subband goes through SVE process, which preserves high frequency components (i.e. edges). Hence, after IDWT, the resultant image will be sharper. In this chapter, the proposed method has been compared with conventional GHE technique as well as LHE and some state-of-art technique such as DHE an SVE. The results indicate the superiority of the proposed method over the aforementioned methods.

\section{The proposed image equalization technique}

There are two significant parts of the proposed method. The first one is the use of SVD. As it was mentioned, the singular value matrix obtained by SVD contains the illumination information. Therefore, changing singular values will directly affect the illumination of the image hence the other information in the image will not be changed. The second important aspect of this work is the application of DWT. As it was mentioned in the introduction, the illumination information is embedded in LL subband. The edges are concentrated in other subbands (i.e. LH, HL, and HH). Hence, separating the high frequency subbands and 
applying the illumination enhancement in LL subband only, will protect the edge information from possible degradation (Demirel et al., IEEE Geoscience and Remote Sensing Letter, 2010). After reconstructing the final image by using IDWT, the resultant image will not only be enhanced with respect to illumination, but also it will be sharper.

The general procedure of the proposed technique is as follows. The input image, $A$, is first processed by using GHE to generate $\hat{A}$. Then both of these images are transformed by DWT into four subband images. The correction coefficient for singular value matrix is calculated by using the following equation:

$$
\zeta=\frac{\max \left(\Sigma_{L L_{\hat{A}}}\right)}{\max \left(\Sigma_{L L_{A}}\right)}
$$

where $\Sigma_{L L_{A}}$ is the LL singular value matrix of the input image and $\Sigma_{L L_{\hat{A}}}$ is the LL singular value matrix of the output of the GHE. The new LL image is composed by:

$$
\begin{aligned}
& \bar{\Sigma}_{L L_{A}}=\zeta \Sigma_{L L_{A}} \\
& \overline{L L_{A}}=U_{L L_{A}} \bar{\Sigma}_{L L_{A}} V_{L L_{A}}
\end{aligned}
$$

Now the $\overline{L L_{A}}$ and $L H_{A}, H L_{A}$, and $H H_{A}$ subband images of the original image are recombined by applying IDWT, to generate the resultant equalized image $\bar{A}$.

$$
\bar{A}=I D W T\left(\overline{L L}_{A}, L H_{A}, H L_{A}, H H_{A}\right)
$$

In this chapter we have used $\mathrm{db} .9 / 7$ wavelet function as the mother function of the DWT. In the following section the experimental results and the comparison of the aforementioned conventional and state-of-art techniques are discussed. Fig. 1 illustrates all steps of the proposed image equalization technique.

\section{Experimental results and discussions}

Fig. 2 (a) illustrates a low contrast image which has been used in (Abdullah Al Wadud et al., 2007). This image has been equalized by using GHE (b), SVE (c), DHE (d), LHE (e), and the proposed equalization technique (f). The quality of the visual results indicates that the proposed equalization technique is sharper and brighter than the one achieved by DHE, GHE, and LHE. The resultant image generated by SVE is comparable with the image achieved by the proposed method.

In order to show the superiority of the proposed method over the SVE, GHE and LHE, Fig 3, and Fig. 4 have been generated. Both figures are showing that the proposed method have a brighter and sharper output, also there is no wash out problem as it has occurred in Fig. 3 and 4 (c) obtained by SVE and there is also no blurring effect like Fig. 3 and 4 (e) obtained by LHE. 


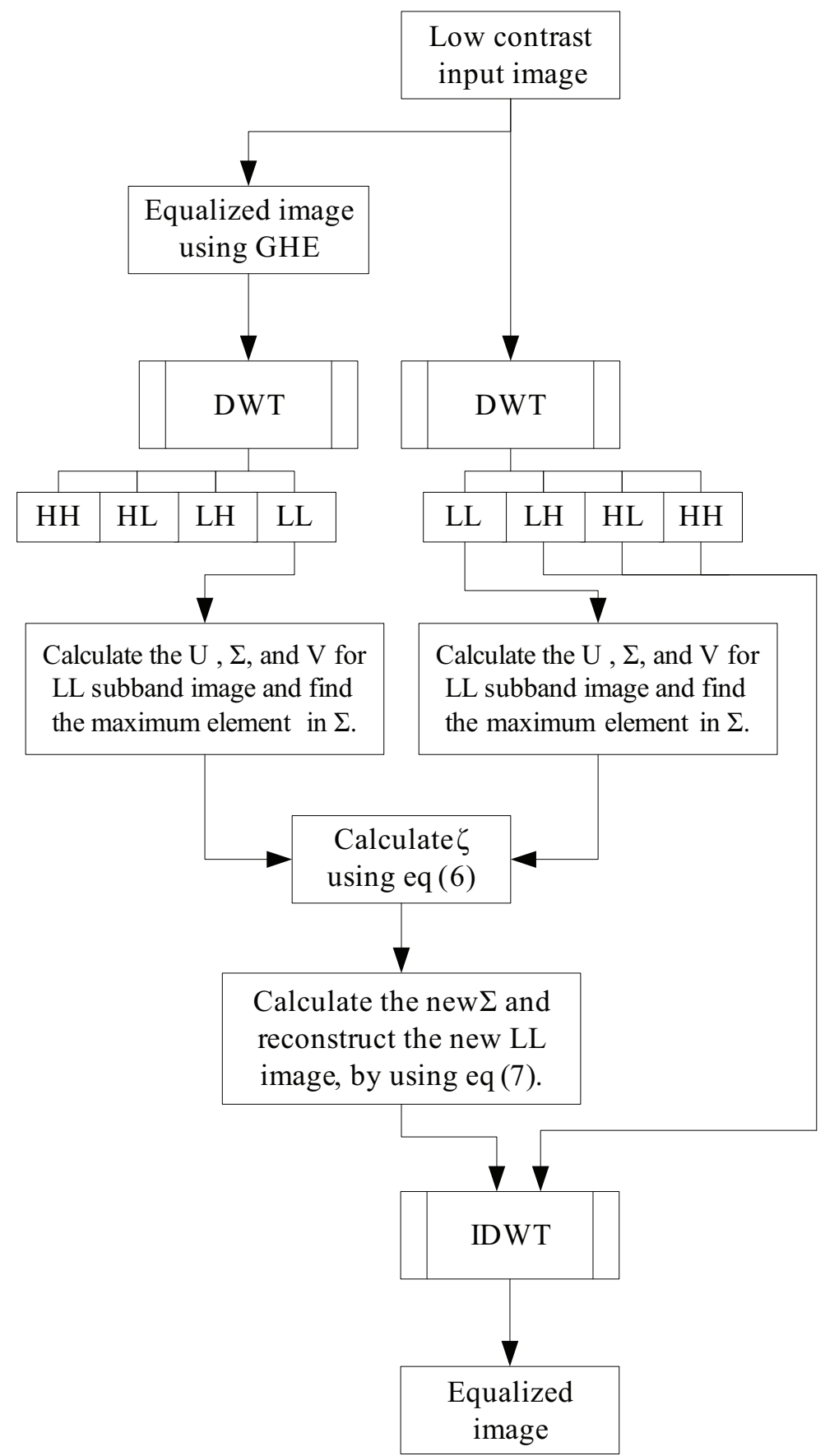

Fig. 1. The detailed steps of the proposed equalization technique. 


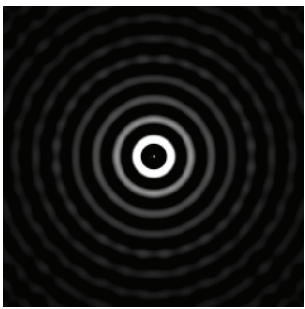

(a) Original

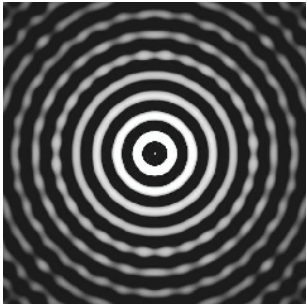

(d) DHE

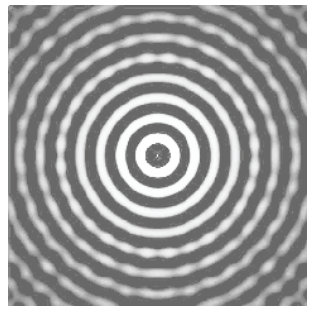

(b) GHE

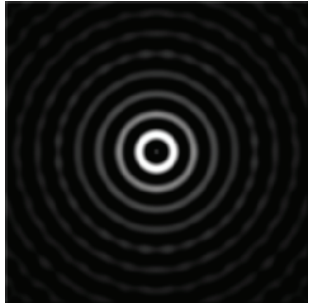

(e) LHE

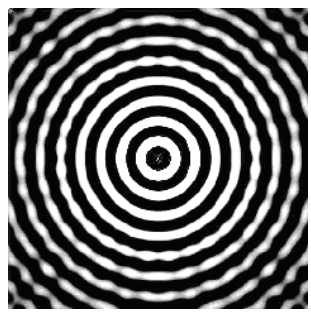

(c) SVE

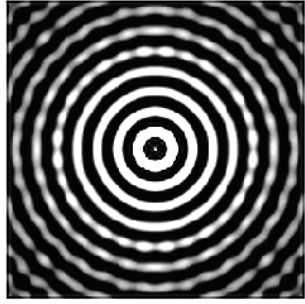

(f) Proposed Technique

Fig. 2. (a) Low contrast image, Equalized image using: (b) GHE, (c) SVE, (d) DHE, (e) LHE, and (f) the proposed technique.

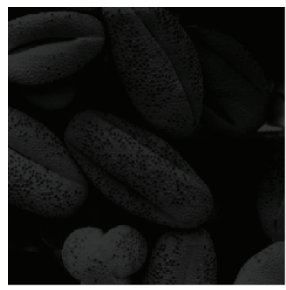

(a) Original

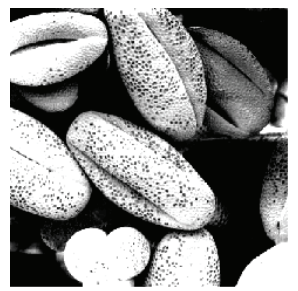

(c) SVE

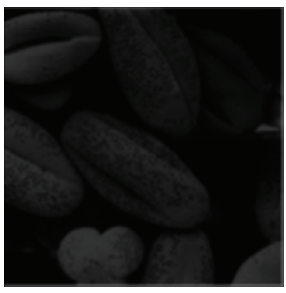

(d) LHE

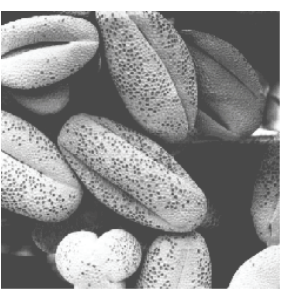

(b) GHE

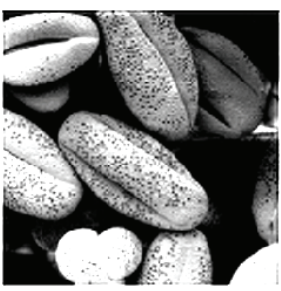

(e) Proposed Technique

Fig. 3. (a) Low contrast image, Equalized image using: (b) GHE, (c) SVE, (d) LHE, and (e ) the proposed technique. 


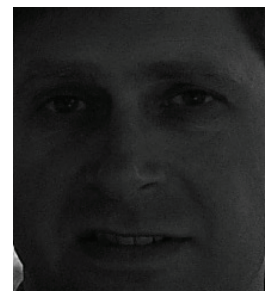

(a) Original

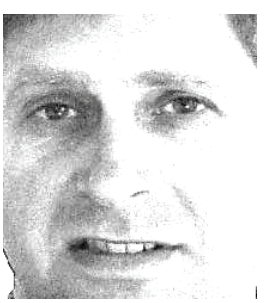

(c) SVE

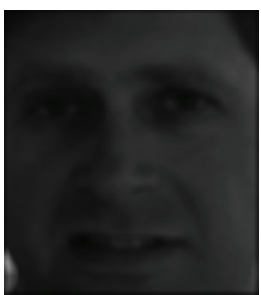

(d) LHE

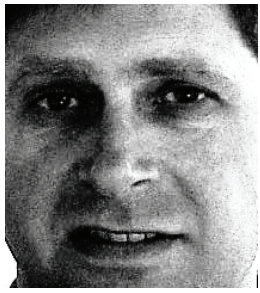

(b) GHE

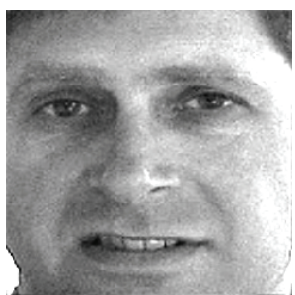

(e) Proposed Technique

Fig. 4. (a) Low contrast image, Equalized image using: (b) GHE, (c) SVE, (d) LHE, and (e ) the proposed technique.

\section{Conclusions}

In this work, a new image equalization technique based on SVD and DWT was proposed. The proposed technique converted the image from spatial domain into the DWT domain and after equalizing the singular value matrix of the LL subband image, it reconstructed the image in the spatial domain by using IDWT. The technique was compared with the GHE, LHE, DHE and SVE techniques. The experimental results were showing the superiority of the proposed method over the conventional and the state-of-art techniques.

\section{Acknowledgements}

Authors would like to thank Haidi Ibrahim and Nicholas Sia Pik Kong from School of Electrical and Electronic Engineering, Universiti Sains Malaysia for providing the equalized output images of DHE technique.

\section{References}

A. R. Weeks, L. J. Sartor, and H. R. Myler, 'Histogram specification of 24-bit color images in the color difference (C-Y) color space', Proc. SPIE, 1999, 3646, pp. 319-329.

C. C. Liu, D. Q. Dai, and H. Yan, "Local discriminant wavelet packet coordinates for face recognition", Journal of Machine Learning Research, 2007, Vol. 8, pp: 1165-1195.

C. C. Sun, S. J. Ruan, M. C. Shie, and T, W. Pai, "Dynamic contrast enhancement based on histogram specification", IEEE Transactions on Consumer Electronics, Vol. 51, No. 4, 2005, pp.1300-1305. 
D. Q. Dai and H. Yan, “Wavelet and face recognition", Face recognition, Chapter 4, K. Delac and M. Grgic (Eds), ISBN: 978-3-902613-03-5, Austria, pp: 59-74, 2007.

H. Demirel, G. Anbarjafari, and M. N. S. Jahromi, "Image equalization based on singular value decomposition", 23 ${ }^{\text {rd }}$ IEEE International Symposium on Computer and Information Sciences, Turkey, Oct 2008, pp. 1-5.

H. Demirel and G. Anbarjafari, "Pose invariant face recognition using probability distribution function in different color channels", IEEE Signal Processing Letter, Vol. 15, 2008, pp. 537 - 540.

H. Demirel, C. Ozcinar, and G. Anbarjafari, "Satellite Image Contrast Enhancement Using Discrete Wavelet Transform and Singular Value Decomposition", IEEE Geoscience and Remote Sensing Letter, April 2010, Vol. 7, No. 2, pp. 334-338.

J. L. Starck, E. J. Candes, and D. L. Donoho, “The curvelet transform for image denoising", IEEE Transactions on Image Processing, 2002, Vol. 11, pp: 670-684.

J. W. Wang and W. Y. Chen, "Eye detection based on head contour geometry and wavelet subband projection", Optical Engineering, 2006, Vol. 45, No. 5.

M. Abdullah-Al-Wadud, H. Kabir, M. A. A.Dewan, C. Oksam, “A dynamic histogram equalization for image contrast enhancement", IEEE Transaction on Consumer Electronics, Vol. 53, No 2, May 2007, pp. 593-600.

M. Lamard, W. Daccache, G. Cazuguel, C. Roux, and B. Cochener, "Use of a JPEG-2000 wavelet compression scheme for content-based ophtalmologic retinal images retrieval", 27th Annual International Conference of the Engineering in Medicine and Biology Society, IEEE-EMBS 2005, pp: 4010 - 4013.

R. C. Gonzalez, and R. E. Woods, Digital Image Processing, Prentice Hall, ISBN 013168728X, 2007.

S. Chitwong, T. Boonmee, and F. Cheevasuvit, 'Enhancement of colour image obtained from PCA-FCM technique using local area histogram equalization', Proc. SPIE, 2002, 4787, pp. 98-106.

T. K. Kim, J. K. Paik, and B. S. Kang, “Contrast enhancement system using spatially adaptive histogram equalization with temporal filtering," IEEE Transactions on Consumer Electronics, Vol. 44, No. 1, 1998, pp. 82-86.

T. Kim, H.S. Yang, “A Multidimensional Histogram Equalization by Fitting an Isotropic Gaussian Mixture to a Uniform Distribution", IEEE International Conference on Image Processing, 8-11 Oct. 2006, pp. 2865 - 2868.

W. G. Shadeed, D. I. Abu-Al-Nadi, and M. J. Mismar, "Road traffic sign detection in color images", 10th International Conference on Environmental and Computer Science, Vol. 2, Dec 2003, pp. 890-893.

Y. Tian, T. Tan, Y. Wang, and Y. Fang, "Do singular values contain adequate information for face recognition?", Pattern Recognition, Vol. 36, 2003, pp. 649 - 655. 


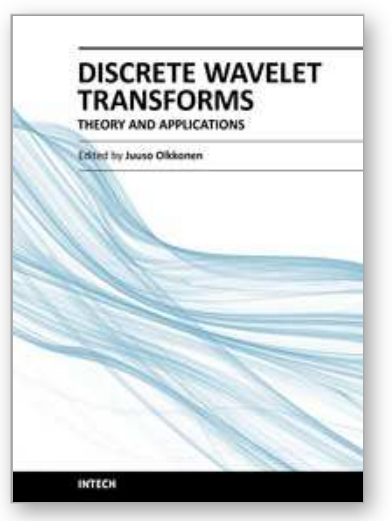

\author{
Discrete Wavelet Transforms - Theory and Applications \\ Edited by Dr. Juuso T. Olkkonen
}

ISBN 978-953-307-185-5

Hard cover, 256 pages

Publisher InTech

Published online 04, April, 2011

Published in print edition April, 2011

Discrete wavelet transform (DWT) algorithms have become standard tools for discrete-time signal and image processing in several areas in research and industry. As DWT provides both frequency and location information of the analyzed signal, it is constantly used to solve and treat more and more advanced problems. The present book: Discrete Wavelet Transforms: Theory and Applications describes the latest progress in DWT analysis in non-stationary signal processing, multi-scale image enhancement as well as in biomedical and industrial applications. Each book chapter is a separate entity providing examples both the theory and applications. The book comprises of tutorial and advanced material. It is intended to be a reference text for graduate students and researchers to obtain in-depth knowledge in specific applications.

\title{
How to reference
}

In order to correctly reference this scholarly work, feel free to copy and paste the following:

Cagri Ozcinar, Hasan Demirel and Gholamreza Anbarjafari (2011). Image Equalization Using Singular Value Decomposition and Discrete Wavelet Transform, Discrete Wavelet Transforms - Theory and Applications, Dr. Juuso T. Olkkonen (Ed.), ISBN: 978-953-307-185-5, InTech, Available from:

http://www.intechopen.com/books/discrete-wavelet-transforms-theory-and-applications/image-equalizationusing-singular-value-decomposition-and-discrete-wavelet-transform

\section{INTECH}

open science | open minds

\author{
InTech Europe \\ University Campus STeP Ri \\ Slavka Krautzeka 83/A \\ 51000 Rijeka, Croatia \\ Phone: +385 (51) 770447 \\ Fax: +385 (51) 686166 \\ www.intechopen.com
}

\author{
InTech China \\ Unit 405, Office Block, Hotel Equatorial Shanghai \\ No.65, Yan An Road (West), Shanghai, 200040, China \\ 中国上海市延安西路65号上海国际贵都大饭店办公楼 405 单元 \\ Phone: +86-21-62489820 \\ Fax: +86-21-62489821
}


(C) 2011 The Author(s). Licensee IntechOpen. This chapter is distributed under the terms of the Creative Commons Attribution-NonCommercialShareAlike-3.0 License, which permits use, distribution and reproduction for non-commercial purposes, provided the original is properly cited and derivative works building on this content are distributed under the same license. 